\title{
Management of Complications of Arteriovenous Grafts in Patients with Chronic Renal Failure
}

\author{
Mohamed Abd El Hamid Abd El Rahman, Islam Taha Ghalwash, \\ Mohammed Abou Al-azayem El-Kenany
}

\author{
Vascular Surgery Department, Faculty of Medicine, Al-Azhar University \\ Corresponding author: Mohammed Abou Al-azayem El-Kenany, E-Mail: dr.m.elkenany@gmail.com, Phone: +0201144277879
}

\begin{abstract}
Background: With increasing number of patients sustained on chronic Haemodialysis, access related surgery forms a significant proportion of vascular surgical practice today. A cursory review of patients with Arteriovenous graft access reveals a considerable variety in short and long term complications that could happen and in the management of those complications.

Aim: The aim of this work is to assess the outcomes of different modalities of management of complications of Arteriovenous grafts in chronic renal failure patient on regular hemodialysis from arteriovenous graft.

Patients and Methods: Thirty chronic renal failure patients with complicated Arterio-venous grafts performed before for regular hemodialysis were enrolled into this study. The patients were recruited from the Vascular Surgery outpatient clinic of El-Hussein University Hospital. Patients were followed up at intervals of: immediately after fistula creation, first day after creation, 1 month, 3 months, 6 months and 9 months after creation for detecting any complications. Follow up of all patients was based on clinical examination and also by duplex ultrasound and CT venography if needed.

Results: Arteriovenous graft complications incidence were higher in male than females, those results were explained by male cases being associated with more co-morbidities and smoking. Venous outflow stenosis and venous hypertension were the most encountered complication, while surgical management in form of ligation and excision was the commonest management modality used. We also noticed a correlation between the duration from creation of AV synthetic graft and interval between primary and secondary intervention. The older the AV graft the longer was the interval between primary and secondary intervention. This can be correlated to years of education those group of patients had, as those patients tended to pay more attention to early manifestations and used better dialysis centers.

Conclusion: Surgical management in form of graft ligation and excision or surgical revision seems to be the simplest yet most effective management modality facing the majority of the complications not neglecting the role of endovascular intervention in venous hypertension.
\end{abstract}

Keywords: ESRD, Hemodialysis, complications, management, surgical, endovascular.

\section{INTRODUCTION}

Arteriovenous Grafts have a relatively short life span if compared with native A-V fistulas and are prone usually to many complications, requiring multiple salvage procedures to maintain their patency. Arteriovenous graft complications are categorized as Thrombotic Complications of A-V access include (Venous Outflow Stenosis and Arterial Inflow Stenosis) and Non-Thrombotic Complications of A-V access include (Bleeding, Pseudoaneurysms, seroma, Arterial steal, Venous hypertension, Neuropathy and Cardiopulmonary complications) ${ }^{(1)}$.

Inherently, dialysis access initiates intimal hyperplasia in the venous outflow. Intimal hyperplasia occurs primarily at the outflow anastomosis of a prosthetic access. It can also involve distant central veins, even in the absence of previous indwelling catheters. Some patients develop intractable intimal hyperplasia; others develop an equilibrium that stabilizes over time. The mechanisms behind arteriovenous (AV) access intimal hyperplasia are ill-defined, but the resulting lesions are common, occurring in the majority of patients with dialysis access making intimal hyperplasia the greatest unsolved problem in Haemodialysis causing Venous Outflow Stenosis or Arterial Inflow Stenosis which will eventually lead to Recirculation and Arteriovenous graft failure or failure to mature representing the thrombotic complication of arteriovenous grafts ${ }^{(2)}$.

Interventions for such Thrombotic complications include either open Surgical Techniques, Percutaneous Techniques, Hybrid Approach or Thrombolysis. Arteriovenous graft accesses tend to thrombose more frequently than autogenous accesses, but because of the presence of $\mathrm{IH}$ at the venous end, they can often be treated repeatedly and provide long-term access without a significant interruption in dialysis. Grafts tolerate balloon catheter thrombectomy better than autogenous accesses because they do not have viable endothelium subject to injury. Segments of the graft can also be replaced as necessary to deal with graft degeneration or even localized soft tissue infections, maintaining access through the residual unaffected graft ${ }^{(3)}$. 
Management of the non-thrombotic Complications of A-V grafts also falls in the same categories as thrombotic ones either Open Surgical Techniques, Percutaneous Techniques, Hybrid Approach or Thrombolysis. Although combination between two different complications or more would be more challenging ${ }^{(4)}$.

\section{AIM OF THE WORK}

The aim of this work is to assess of the outcomes of different of modalities of management of complications of Arterio-venous grafts performed before for regular hemodialysis in patients with chronic renal failure placed at a single institution during a 9 months' period and document any salvage procedures done to maintain and prolongs arteriovenous grafts patency.

\section{PATIENTS AND METHODS}

This is a retrospective study of the management of arteriovenous graft complication in our hospital (El-Hussein University Hospital). The inpatient medical charts of the patients spanning 9 months (Oct 2017 to July 2018) were reviewed. Data were obtained on their demographic, clinical, management and outcome parameters.

This study was conducted on thirty end stage renal disease (ESRD) patients who were presented at AL Hussein University Hospital in Cairo with different arteriovenous graft complications. The study group included patients from both sex.

Patient under 18 years old, pregnant females, patients enrolled for renal transplantation, patients with native $\mathrm{A}-\mathrm{V}$ fistula or patients with temporary or permanent dialysis catheters were excluded.

All patients underwent full history taking, clinical examination, Duplex examination for $\mathrm{A}-\mathrm{V}$ graft functionality, CT contrast imaging if needed, culture and sensitivity in case of infection and of course any surgical, endovascular or combined interventions in management for any complication would be documented.

All patients underwent follow up starting from day zero of creation of the A-V synthetic graft fistula, first day, then at intervals of 1 month, 3 months, 6 months and 9 months. Intervals between primary and secondary interventions in cases that needed reintervention were also recorded and considered.

\section{Ethical and approval statements}

Informed written consents were obtained from all patients. The approval from the research ethics committee of the faculty of medicine, AlAzhar University Hospitals was also obtained.

\section{Statistical analysis}

Data were statistically described in terms of mean \pm standard deviation $( \pm \mathrm{SD})$, median and range, or frequencies (number of cases) and percentages when appropriate. Comparison of numerical variables between the study groups was done using Student ttest for independent samples in comparing 2 groups, and one way analysis of variance (ANOVA) test with post-hoc multiple 2 group comparisons when comparing more than 2 groups. For comparing categorical data, Chi-square $(\chi 2)$ test was performed. Exact test was used instead when the expected frequency is less than 5. Correlation between various variables was done using Pearson moment correlation equation for linear relation in normally distributed variables and Spearman rank correlation equation for non-normal variables/ non-linear monotonic relation. "p" values less than 0.05 was considered statistically significant. All statistical calculations were done using computer program IBM SPSS (Statistical Package for the Social Science; IBM Corp, Armonk, NY, USA) release 22 for Microsoft Windows.

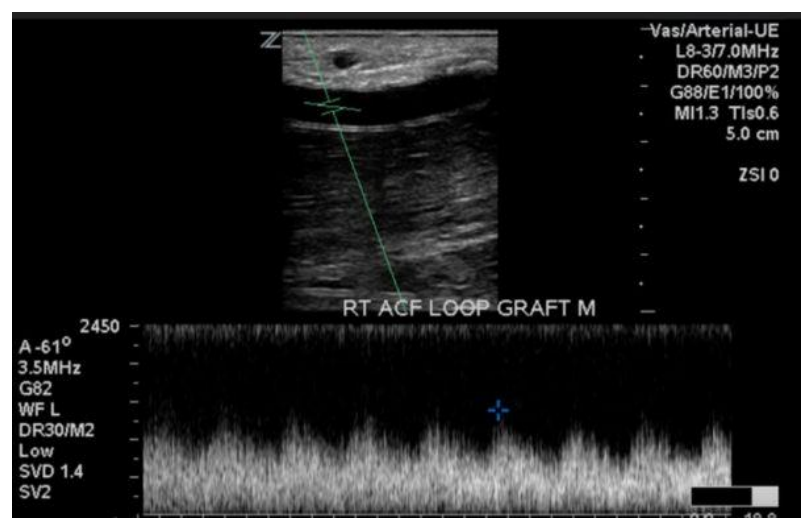

Figure (1): Duplex study of loop forearm A-V graft.

\section{RESULTS}

In our study which was conducted on thirty patients we had found that $63.33 \%$ of the patients were males and $36.67 \%$ were females. males had a mean age of $55.37 \pm 8.43$ years, they started to develop complications in a period of $5.27 \pm 2.47$ months from creation of their fistula, while females had a mean age of $51.63 \pm 8.87$ years and started to develop complications after $6.36 \pm 1.82$ months from creation of their fistulas. 
$21.1 \%$ of the male patients were diabetic $10.5 \%$ were hypertensive only, $36.8 \%$ where diabetic and hypertensive and $52.6 \%$ were smokers.

As for females, $18.2 \%$ had diabetes, $45.5 \%$ were hypertensive, $9.1 \%$ were diabetic and hypertensive and only $9.1 \%$ were smokers.

Table (1): Co-morbidities in both males and females.

\begin{tabular}{|l|c|c|c|}
\hline Co-morbidities & $\begin{array}{c}\text { Males } \\
(\mathbf{N = 1 9 )}\end{array}$ & $\begin{array}{c}\text { Females } \\
(\mathbf{N}=\mathbf{1 1})\end{array}$ & $\begin{array}{c}\text { Total } \\
(\mathbf{N}=\mathbf{3 0})\end{array}$ \\
\hline No & $6(31.6 \%)$ & $6(31.6 \%)$ & $9(30 \%)$ \\
\hline Diabetes & $4(21.1 \%)$ & $4(21.1 \%)$ & $6(20 \%)$ \\
\hline HTN & $2(10.5 \%)$ & $2(10.5 \%)$ & $7(23.3 \%)$ \\
\hline HTN and DM & $7(36.8 \%)$ & $7(36.8 \%)$ & $8(26.6 \%)$ \\
\hline
\end{tabular}

$93.3 \%$ of the all observied arteriovenous grafts were on the upper limb with $78.6 \%$ of them were upper arm graft, and $21.4 \%$ were forearm grafts. The left side was the most observed side with a percent of $63.3 \%$. Only 2 female patients had thigh graft.

Table (2): Site of the A-V graft fistula in both males and females.

\begin{tabular}{|l|c|c|c|}
\hline Site of fistula & Males $(\mathbf{N}=19)$ & Females $(\mathbf{N}=11)$ & Total $(\mathbf{N}=\mathbf{3 0})$ \\
\hline Right & $6(31.57 \%)$ & $5(45.45 \%)$ & $11(36.66 \%)$ \\
\hline Left & $13(68.42 \%)$ & $6(54.54 \%)$ & $19(63.33 \%)$ \\
\hline Upper limb & $19(100 \%)$ & $9(81.81 \%)$ & $28(93.33 \%)$ \\
\hline Lower limb & $0(0 \%)$ & $2(18.18 \%)$ & $2(6.66 \%)$ \\
\hline
\end{tabular}

The most common complication in all studied patients was venous outflow stenosis and venous hypertension, accounting for $23.3 \%$ of patients. However, bleeding (solely or combined with other complications) was the most common complication in male patients group, representing $42 \%$, while infection (solely or combined) was the most common complication in female patients group, representing $36.4 \%$. Staphylococcus infection was the most common type of infection. S. aureus was the most prevalent with a percent of $50 \%$, followed by Ecoli in $33.3 \%$ and lastly S. epidermidis in $16.6 \%$.

Table (3): Prevalence of organisms across the infected cases

\begin{tabular}{|l|c|c|}
\hline \multicolumn{1}{|c|}{ Organism } & Number of patients & Percent \\
\hline Staphylococcus aureus & 3 & $50 \%$ \\
\hline E.coli & 2 & $33.3 \%$ \\
\hline Staphylococcus epidermidis & 1 & $16.6 \%$ \\
\hline
\end{tabular}

As for management modalities surgical management was the most commonly used option for managing AV synthetic graft complications. Ligation was the commonest management modality in the studied patients (30\% in all patients, $31.6 \%$ in male patients, $27.3 \%$ in female patients). The least used modality of management was stenting $(6.7 \%$ in all patients, $0 \%$ in male patients, $18.2 \%$ in female patients).

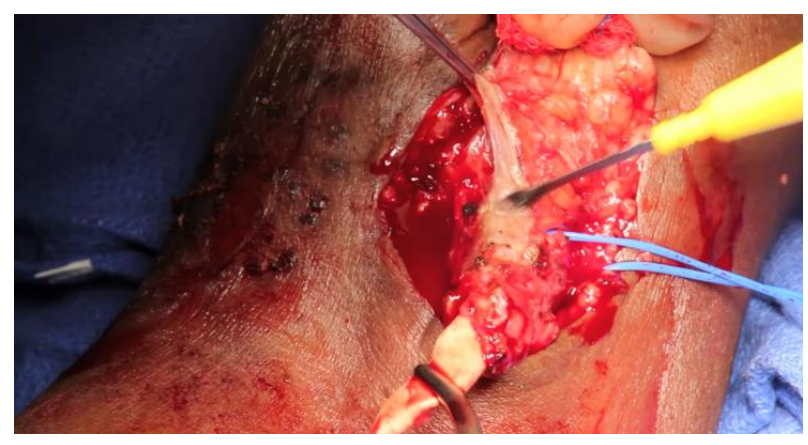

Fig (2): A-V graft ligation and excision.

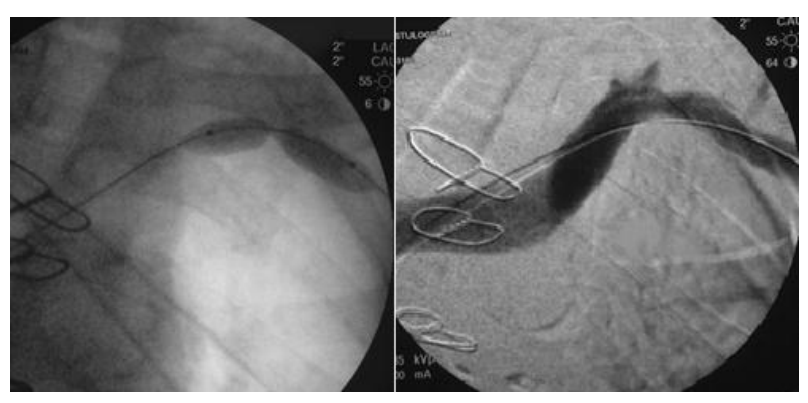

Fig (3): Before and after image of endovascular venous ballooning of an upper arm arteriovenous graft.

\section{DISCUSSION}

Chronic kidney disease (CKD) is increasingly recognized as a public health problem in Egypt and it's holding the 7th cause of death in Egypt after heart diseases, cerebral stroke and cancer. ESRD patient numbers is increasing in Egypt, with annual rate of 74 new patients for each 1 million of the population. And the total number of patients receiving hemodialysis is 264 patients for each 1 million of population. So Maintenance of hemodialysis is our major challenge as renal transplantation is a lengthy and difficult procedure either due to large number of ESRD patients or due to lack and restrictions over kidney donations. ${ }^{(5)}$

Currently the hemodialysis therapy is available in almost each major governmental hospital in Egypt, but the costs for those kind of therapy are still a burden. ${ }^{(6)}$

Derakhshanfar and his colleagues ${ }^{(7)}$ reported that the most frequently encountered complication in the vascular access was Pseudoanyrism (51\%) followed by venous hypertension (16.7\%), infection (4.4\%), thrombosis $(3.3 \%)$ and arterial steal $(1.1 \%)$ and for infection complication Ratnaja and Susan ${ }^{(8)}$ reported 
that the prevalence of $\mathrm{S}$. aureus in patients on hemodialysis is (35-62\%). In the study of Astor and his coworkers ${ }^{(9)}$, the prevalence of Staphylococcus aureus (S. aureus) nasal carriage in patients on hemodialysis is (35-62\%), a factor that might predispose these patients to $S$. aureus.

Morsy et al. ${ }^{(10)}$ reported that arterial steal was cured by Banding in (90 - 100\%) of a group of 6 patients, but only $(10-40 \%)$ of the banded accesses remained patent. Schanzer et al. ${ }^{(11)}$ reported a success rate of (93\%) with DRIL in a group of 14 patients and (83\%) of the accesses remain patent.

Beathard $\boldsymbol{e t}$ al. ${ }^{(12)} 58$ patients with prosthetic $\mathrm{AV}$ accesses and venous anastomotic stenosis were randomized to PTA or PTA with an adjunctive stent. No significant difference was noted in access thrombosis, need for surgical revision, or need for a repeat PTA. In yet another trial, 34 patients with stenosis at or $<3 \mathrm{~cm}$ of the outflow vein-prosthetic graft junction or in the peripheral outflow vein were randomized to PTA or PTA with an adjunctive stent. Hoffer et al. (13) concluded that stent placement offered no advantage.

\section{CONCLUSION}

Management of arteriovenous graft complication was and still a major part of the daily practice on any vascular surgeon, Loss of hemodialysis arteriovenous vascular access is the major end result of a lot of complications which can be avoided or solved if proper surveillance and management procedures were taken. Surgical management in forms of ligation or revision surgery is still superior to endovascular management in the majority of the cases. As it offers simple yet effective means to eliminate and encounter the complication.

In our study we also notice a correlation between the duration from creation of AV synthetic graft and interval between primary and secondary intervention, the older the AV graft the longer was the interval between primary and secondary intervention. This can be correlated to years of education those group of patients had, as those patients tended to pay more attention to early manifestations and used better dialysis centers.

\section{REFERENCES}

1. Miller PE, Carlton D, Deierhoi MH et al. (2000): Natural History of Arteriovenous Grafts in Hemodialysis Patients. Am J Kidney Dis., 36(1):68-74.
2. Altman SD (2007): A practical approach for diagnosis and treatment of centralvenous stenosis and occlusion. SeminVasc Surg., 20:189-194.

3. Sprouse LR, Lesar CJ, Meier GH et al. (2004): Percutaneous treatment of symptomatic central venous stenosis (corrected). J Vasc Surg., 39:578-582.

4. Schanzer H (2002): Overview of complications and management after vascular access creation. Gray RJ, ed. Dialysis Access. Philadelphia: Lippincott Williams \& Wilkins.

5. Ahmed R El-Arbagy, Yassin $S$ Yassin, Boules N Boshra (2016): Study of prevalence of endstage renal disease in Assiut governorate, upper Egypt. Menoufia Med J., 29: 222-7.

6. Soliman AR, Fathy A, Roshd D et al. (2012): The growing burden of end-stage renal disease in Egypt. Renal Failure, 34(4):425-8.

7. Derakhshanfar A, Gholyaf M, Niayesh A et al. (2009): Assessment of frequency of complications of arteriovenous fistula in patients on dialysis: a two-year single center study from Iran. Saudi J Kidney Dis Transplant., 20:872-5.

8. Ratnaja $K$ and Susan $H$ (2007): Central venous catheter-related bacteremia in chronic hemodialysis patients: epidemiology and evidence-based management. Nat Rev Nephrol., 3:256-66.

9. Astor BC, Eustace Joseph A, Powe Neil R et al. (2005): Type of vascular access and survival among incident hemodialysis patients: the choices for healthy outcomes in caring for ESRD (CHOICE) study. J Am Soc Nephrol., 116:1449-55.

10. Morsy AH, Kulbaski M, Chen C et al. (1998): Incidence and characteristics of patients with hand ischemia after a hemodialysis access procedure. J Surg Res., 74(1):8-10.

11. Schanzer H, Skladany M, Haimov M (1992): Treatment of angioaccess-induced ischemia by revascularization. J Vasc Surg., 16: 861-866.

12. Beathard GA (1993): Gianturco self-expanding stent in the treatment of stenosis in dialysis access grafts. Kidney International, 43(4):872-7.

13. Hoffer EK, Sultan S, Herskowitz MM et al. (1997): Prospective randomized trial of a metallic intravascular stent in hemodialysis graft maintenance. Journal of Vascular and Interventional Radiology., 8(6):965-73. 